\title{
BIM-BASED ENERGY MORNITORING WITH XML PARSING ENGINE
}

\author{
Jeong-Han Woo*, Carol Diggelman, and Bass Abushakra \\ Civil and Architectural Engineering and Construction Management Department, \\ Milwaukee School of Engineering, Milwaukee, USA \\ *Corresponding author (woo@msoe.edu)
}

\begin{abstract}
Due to the urgent need for a response to energy security, aging commercial buildings are required to conform to increasingly stringent environmental standards, particularly with regard to energy and water consumption. Solutions to reach the energy-related goals seem to exist. However, it is often difficult to gain synergistic benefits from the installations. The energy savings actually achieved are often disappointing. Systematic measurement and verification are rarely available due to the high initial cost of such studies. Therefore, there is a significant chance that misunderstandings and unrealistic expectations will affect decision making related with building sustainability. The ability to synthesize emerging technologies into a robust computational platform for monitoring coupled human-building environments is lacking. This paper discusses developing a prototype of BIM-based Baseline Building Model (B3M) for aging commercial buildings. As-built BIM of a public building was employed as a platform for delivering user-actionable information to building occupants, maintaining healthy environments, and achieving further energy savings. Initial testing was conducted at the Technology Innovation Center with data collected for four months. The results of this study showed that B3M provides a robust computational platform for monitoring coupled human-building environments. B3M would be a useful system when aging commercial buildings need to measure and verify accurate benefits of the installations.
\end{abstract}

Keywords: BIM, Energy Efficiency, Baseline Building Model, XML Parser, Sensor Network

\section{BIM-BASED BUILDING ENERGY MODEL}

Building Information Modeling (BIM) is one of the most significant recent innovations in architecture, engineering and construction. It integrates the description of static building data such as 3D geometries, materials, building structures, Mechanical, Electrical, and Plumbing (MEP) systems, occupants, etc. into an intelligent format. The use of BIM has been focused on building design rather than building operations and facility management. Although the potential of using as-built BIM for facility management is well known, a systematic approach to provide the necessary information for sustainable buildings is yet to be developed [1].

Expanded use of BIM would allow for more detailed analysis of building energy performance. BIM-based building energy models can be designed to store energy performance data such as consumption, temperature, $\mathrm{CO} 2$ emissions, occupancy, and humidity. In addition, the connection to the BIM allows facility managers to simulate occupancy changes, facility upgrades, or energy management strategies based on potential energy demands [2].

If a building baseline model is created directly from an upto-date BIM, the energy model can be regenerated as the operating conditions are updated. Facility management processes can be improved significantly through the use of an as-built BIM that can integrate real-time building performance data.

\section{AS-BUILT BIM}

The Technology Innovation Center (TIC) of the Milwaukee County Research Park (MCRP) was used as a test bed. Data from the sensors were recorded on Onset's HOBO ${ }^{\circledR}$ Ethernet Communications Data Loggers, model U30-ETH, in 5-minute intervals and were transmitted to the Onset's database server via data organization software HOBOware 
Pro. Examples of output from the system can be seen at the web-site:

https://www.hobolink.com/p/691f87a78e34150be0d0a0f90 5 f0c6b6. The equipments were in-stalled and calibrated on January 22, 2010. Some minor problems were identified and fixed by April 14, 2010. An example of monitored energy consumption data is shown in Figure 1.

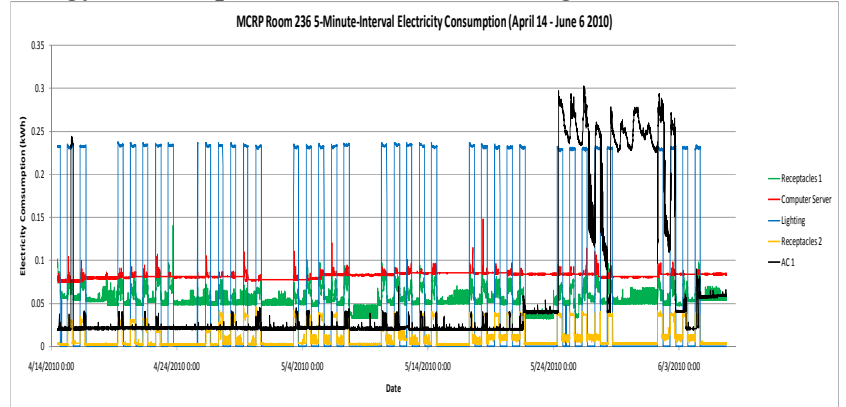

Fig. 1 Electricity Consumption of Room 2

Building data from the as-built BIM were unified with monitored data to provide accurate building baseline conditions. As-built BIM was created based on 3D point clouds data as shown in Figure 2.

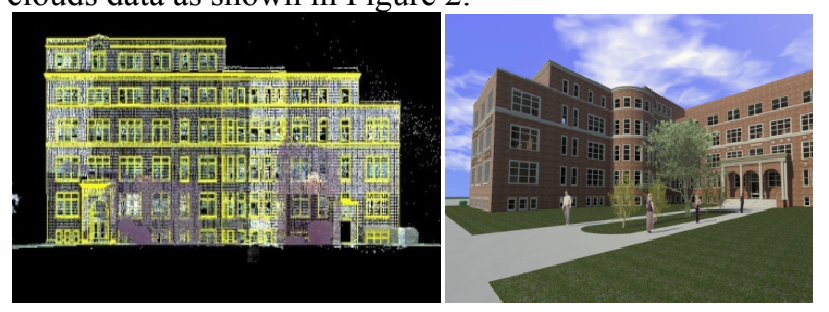

Fig. 2 Point Clouds and As-built BIM

\section{XML PARSING ENGINE}

The datasets are delivered in SensorML, an industry standard XML schema, defining the geometric, dynamic, and observational characteristics of sensors. A parsing engine was developed to translate SensorML monitored data to be used in a .NET and Revit API environment. The Parsing Engine, called MCRP PRO, is designed to access to the Onset's database server to extract recorded sensor data. The functions of the Parsing Engine are as follows: receives data, decrypt, parse, send to data grid view, and insert data into MS Access database. MCRP PRO was developed using .NET programming languages, including Visual Basic and C\#. Visual Studio.NET was chosen as the development platform to establish the internet-based application via ODBC protocol (Figure 3).

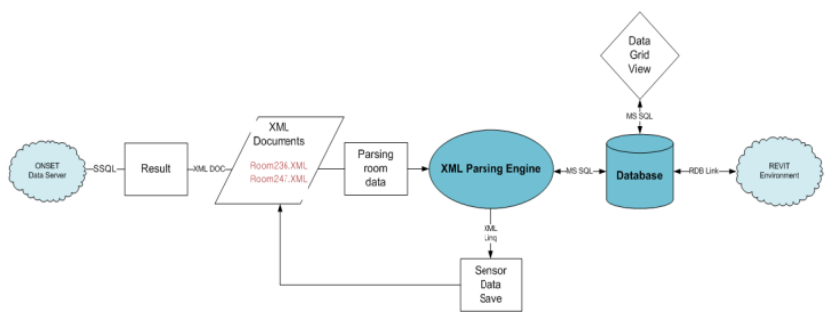

Fig. 3 Concept of XML Parsing Engine

The parsing engine used pre-defined algorithms to parse the data and to send and receive data from different data protocols, normalize the data, and exchange it in an XML format. It also converts the SensorML data to open $\mathrm{XML} / \mathrm{SOAP}$ Services so that it can be used in .NET environment. Finally, it mapped data to .NET applications which store the data into the database. Figure 4 shows the user interface of MCRP Pro.

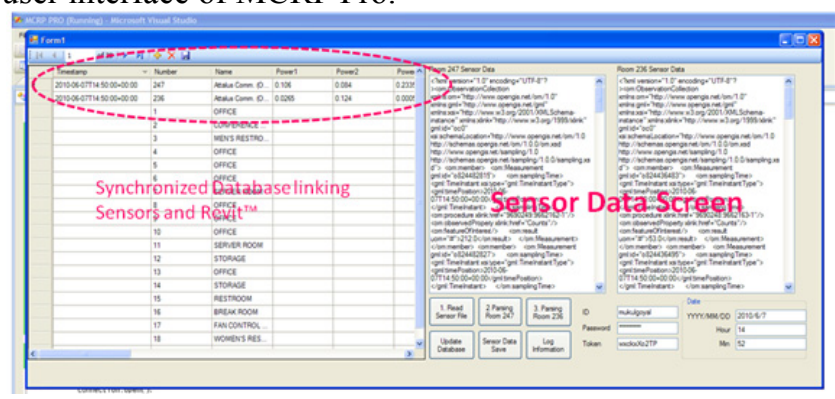

Fig. 1 MCRP Pro

\section{CONCLUSION}

B3M would be a useful system when aging commercial buildings need to measure and verify accurate benefits of the installations. The integrated data framework has a potential to create substantially more interconnected building systems and reduce operational and environmental risks. The future research will develop a set of exemplary B3M-based applications to test and verify its ability to meet user needs.

\section{REFERENCES}

[1] Young, N., Jones S., and Bernstein H., The Business Value of BIM, New York: McGraw Hill, 2009.

[2] Granade, H., et al., Unlocking Energy Efficiency in U.S. Economy. McKinsey \& Company, 2009. 